\title{
Terahertz plasmonics: achievements and prospects
}

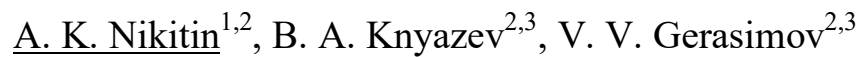 \\ ${ }^{1}$ Scientific and Technological Center of Unique Instrumentation, Russian Academy of Sciences, Moscow, Russia, \\ alnikitin@mail.ru \\ ${ }^{2}$ Novosibirsk State University, Novosibirsk, Russia \\ ${ }^{3}$ Budker Institute of Nuclear Physics, Siberian Branch of Russian Academy of Sciences, Novosibirsk, Russia
}

\section{Brief theory and definitions}

Plasmons are collective oscillations of the free electron gas density. Just as light consists of photons, the plasma oscillation consists of plasmons. Plasmon coupled with photon forms another quasiparticle called a plasmon-polariton. Plasmon-polaritons propagating along a "metal-dielectric" interface are called surface plasmon-polaritons (SPPs). They represent themselves a complex of an evanescent $p$-polarized electromagnetic wave coupled with a wave of density of free charges on surface of a (semi) conductor.

The field of optics studying surface plasmons is often described as Plasmonics, by analogy with electronics [1]. "SPPs is the best known method for confining and guiding EM radiation into the smallest possible volume" [2].

By solving Maxwell's equations with the appropriate boundary conditions, one can find out that: 1) field of surface plasmons is $p$-polarized, in order to build polarization of the metal surface; 2) they can exist provided the metal dielectric constant is negative; 3 ) their dispersion relation (between the tangential component of the SPP wave number $k_{x}$ and the angular frequency $\omega$ of their field) for a "metaldielectric" interface has the form [2]:

$$
k_{x}=\frac{\omega}{c} \cdot \sqrt{\frac{\varepsilon_{\mathrm{M}} \cdot \varepsilon_{\mathrm{D}}}{\varepsilon_{\mathrm{M}}+\varepsilon_{\mathrm{D}}}},
$$

here $c$ is the speed of light in free space; $\varepsilon_{\mathrm{M}}$ and $\varepsilon_{\mathrm{D}}$ are the dielectric constants of the metal and the dielectric.

The complex value of $\varepsilon_{\mathrm{M}}$ makes $k_{x}$ a complex quality as well, with a real component $k_{x}^{\prime}$ that defines the dispersion of SPPs and an imaginary component $k_{x}^{\prime \prime}$ that is proportional to the Joule losses of the SPPs.

The dispersion curves for SPPs on a metal adjoining vacuum or a dielectric are presented in Fig.1. The dotted lines indicate so called "light lines" depicting the dispersion of plane waves in a relevant medium. As one can see, the real component of the SPP wave number is close to the light lines at low frequencies; SPPs have light-like characteristics in the terahertz $(\mathrm{THz})$ range that is $\mathrm{THz}$ SPPs are weakly bounded to the interface and propagate a long distance (meters). The value of $k_{x}^{\prime}$ deviates from the respective light line as the frequency increases, which is accompanied by stronger confinement (in the dielectric) of the SPPs to the interface. Note, that as $\omega$ approaches the value $\omega_{S P P}=\omega_{p} / \sqrt{1+\varepsilon_{D}}$ (here $\omega_{p}$ is the plasma frequency of the metal) the value of $\varepsilon_{M} \rightarrow 0$ and the deviation of $k_{x}^{\prime}$ from $k_{\mathrm{o}}$ (the wave number of a plane wave in vac- uum) infinitely increases, while the propagation length of the SPPs is drastically reduced [2]. Thus one may state that in the low-frequency limit ( $\mathrm{THz}$ range) SPPs acquire the photonic character, while in the high-frequency limit $\left(\omega \rightarrow \omega_{S P P}\right)$ they resemble a mechanical wave (plasmonic character).

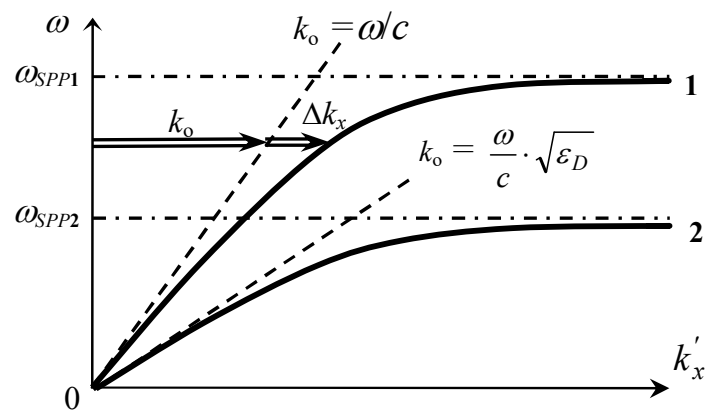

Fig. 1. The dispersion curves of SPPs on interfaces "metal vacuum" (curve 1) and "metal - dielectric with $\varepsilon_{D}$ " (curve 2).

From the expression for $\omega_{S P P}$ it follows that there are at least two ways for controlling confinement (as well as the propagation length) of SPPs to the surface: 1) to change the value of $\omega_{p}$ by, for example, replacing the metal with a semiconductor; 2) to cover the surface with a dielectric layer (curve 2 in Fig.1).

\section{Achievements in THz plasmonics}

Metals have very large permittivity at $\mathrm{THz}$ frequencies, typically 5 to 6 orders of magnitude larger than at optical frequencies. This reduces the penetration depth of the SPP field into the metal and results in a weak confinement of the field to the surface, as well as in an increase of the SPP propagation length $L$ due to the reduction of ohmic loses in the metal.

According to the Drude model for the dielectric permittivity of metals values of $L \sim \lambda^{2}$ (here $\lambda$ is the radiation wavelength). But already first experiments with SPPs excited by $\mathrm{THz}$ gas lasers revealed great discrepancy between the calculated and measured $L$ values. In the experiments, THz SPPs ran distances an order or even two orders smaller than those predicted by the theory: real THz SPPs ran from a few to tens of centimeters [3]. As it was found out later, the main reason for this was not only the difference between the dielectric permeability of the metal surface and its bulk value, but also the significant radiative losses arising from scattering of SPPs on the surface inhomogeneities [4]. To reduce the losses it was suggested to cover the surface with a thin $(\sim \lambda / 100)$ dielectric layer shifting in this way the dispersion curve from the light line and thus making the "SPPs - bulk radiation" process less probable. 
Later on it was proposed to employ the newly developed time-domain spectroscopy (TDS) technique for experimental studies of THz SPPs $[5,6]$. Using the technique Prof. D. Grischkowsky with colleagues estimated average values of $L$ and SPP field penetration depth $\delta$ into air; they also discovered the capability of THz SPPs to jump through $\mathrm{cm}$ air gaps between the adjacent metal plates. The TDS is beneficial in many respects when investigating bulk samples, but this method has obvious pitfalls of dispersion origin when SPPs are under study. Using monochromatic radiation produced by lasers, one can avoid problems with dispersion of broadband SPPs, uncertainty in phase shifts at the coupling and decoupling elements, as well as the necessity of the inverse Fourier transform in data processing.

Intensive studies of $\mathrm{THz}$ SPPs are conducted with the Novosibirsk free-electron laser (Novo FEL) at the Siberian Synchrotron and Terahertz Radiation Centre [7]. Here they managed to master the end-fire coupling method for effective conversion of FEL radiation into $\mathrm{THz}$ SPPs employing cylindrical segments as coupling elements; this made it possible to measure carefully field distribution along the SPP track, to study diffraction of $\mathrm{THz}$ SPPs on the rectangular sample edge, to detect bulk waves emitted from the SPP track, to investigate explicitly the process of SPP passing from one to another substrate via air gaps, to establish capability of $\mathrm{THz}$ SPPs to be reflected by plane mirrors and to be divided by plane beam splitters $[4,8,9]$. Based on these achievements we have developed and tested the device for measuring the propagation length of $\mathrm{THz}$ SPPs in which the input and output elements are fixed; this enables one to perform measurements with a high signal-to-noise ratio.

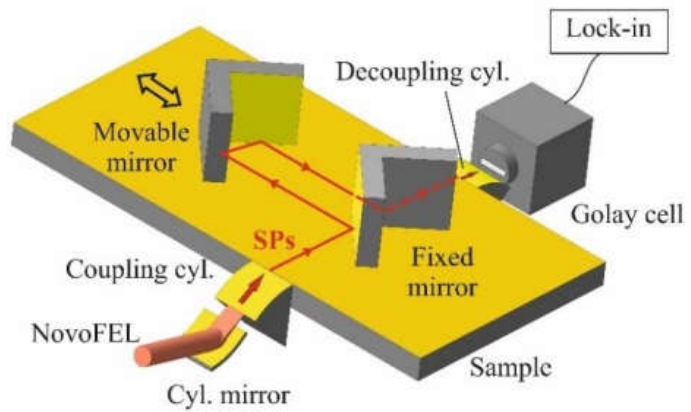

Fig. 2. Device with fixed coupling elements for measuring the propagation length of THz SPPs.

Recently one more way for controlling the value of $\omega_{p}$, and thus the value of $\omega_{S P P}$, was discovered; this method consists in creating periodic structures (holes, recesses, or objects) of subwavelength dimensions on the metal surface [10]. Such SPPs were called "spoof" or "designed" surface plasmons. The method allows confining THz SPPs not only on metals, but also on graphene, as well as on superconductors [1, 2]. Moreover, it turned out that conductive layers "decorated" with ordered holes have the ability to selectively transmit $\mathrm{THz}$ radiation with the transmission efficiency proportional to $(r / \lambda)^{4}$ (here $r$ is the radius of each hole).

\section{Prospects of THz plasmonics}

We can distinguish the following promising areas of THz plasmonics development:

1) SPP communication channels based on extended metal-dielectric structures (wires) with rectangular or circular cross-section. The advantages of this method of information transmission are the following: first, the speed of THz SPPs is the maximum possible (it almost equals the speed of light); secondly, it leads to an extremely low group velocity dispersion, allowing essentially undistorted pulse propagation;

2) design of SPP interferometers that will make it possible to create surface plasmon Fourier spectrometers of the $\mathrm{THz}$ range;

3) intensive study of "spoof" surface plasmons (especially on graphene) and their interaction with bulk $\mathrm{THz}$ radiation;

4) generation of $\mathrm{THz}$ SPPs on semiconductor surfaces by radiation with $\omega \approx \omega_{p}$ condensing the light in the vicinity of the surface and thus paving the way to $\mathrm{THz}$ microscopy with super high resolution;

5) study of "localized" SPPs (LSPPs) in semiconductor micron-size particles which, in contrast to metal ones, are pervious for $\mathrm{THz}$ radiation. Exciting LSPPs one may concentrate the radiation energy in a very limited space.

The work was supported by the Russian Science Foundation (grants No. 18-12-00430 and 14-50-00080).

\section{References}

1. Maier, S. A. Plasmonics: Fundamentals and Applications. Springer, 2007. $-224 \mathrm{p}$.

2. Gomez, R. J., Berrier A. Fundamental aspects of surface plasmon polaritons at THz frequencies // In "Handbook of THz technology“. Eds by Saeedkia D. Woodhead Publ. Series in Electronic and Optical Materials, 2013. - 688 p.

3. Koteles, E. S., McNeill, W. H. Far infrared surface plasmon propagation // Intern. J. Infrared Millim. Waves. 1981. V. 2. P. $361-371$

4. Gerasimov, V.V., Knyazev, B.A., Lemzyakov, A.G., Nikitin, A.K., Zhizhin, G.N. Growth of THz surface plasmon propagation length due to thin-layer dielectric coating // JOSA (B). 2016. V. 33. Is. 11. P. 2196-2203.

5. Jeon, T.-I., Grischkowsky, D. THz Zenneck surface wave (THz surface plasmon) propagation on a metal sheet // Appl. Phys. Lett. 2006. V. 88. 061113.

6. Nazarov, M. M., Mukina, L. S., Shuvaev, A.V., Sapozhnikov, D. A., Shkurinov, A. P., Trofimov, V. A. Excitation of surface EM waves studied by $\mathrm{THz}$ spectrochronography // Laser Phys. Lett. 2005. V. 2, No. 10. P. 471-475.

7. Kulipanov, G. N., Bagryanskaya, E. G., Chesnokov, E. N. et al. Novosibirsk free electron laser - facility description and recent experiments // IEEE Trans. on THz Science and Technology. 2015. V. 5. No. 5. P. 798-809.

8. Gerasimov, V.V., Knyazev, B.A., Nikitin, A.K., Zhizhin, G.N. Experimental investigations into capability of THz surface plasmons to bridge macroscopic air gaps // Optics Express. 2015. V. 23. No. 26. P. 33448-33459.

9. Gerasimov, V.V., Knyazev, B.A., Nikitin, A.K. Reflection of $\mathrm{THz}$ surface plasmon-polaritons by a $\mathrm{fl}$ at mirror // Quantum Electronics. 2017. V. 47. No. 1. P. 65-70.

10. Pendry, J. B., Martín-Moreno, L., García-Vidal, F. J. Mimicking surface plasmons with structured surfaces // Science. 2004. V. 305. No. 5685. P. 847-848. 